\title{
Feasibility of the Gastrografin Challenge for Adhesive Small Bowel Obstruction
}

\author{
Ki-Sang Jung a , Kyoung Jin Choi a , Kyoung Won Yoon a, Keesang Yoo a, Eunmi Gil a,b, Chi-Min Park a,b,* \\ ${ }^{a}$ Department of Surgery, Samsung Medical Center, Sungkyunkwan University of Medicine, Seoul, Korea \\ ${ }^{b}$ Department of Critical Care Medicine, Samsung Medical Center, Sungkyunkwan University of Medicine, Seoul, Korea
}

Article history:

Received: October 19, 2020

Revised: November 5, 2020

Accepted: November 9, 2020

\begin{abstract}
Purpose: This retrospective study investigated the feasibility, diagnostic, and therapeutic advantages of the gastrografin challenge on patients with adhesive small bowel obstruction (ASBO).

Methods: There were 125 patients reviewed who were admitted to the Department of General Surgery at a single institution (September 2018 to August 2019) with a diagnosis related to ASBO. The study population included 100 patients (114 cases) who had received initial conservative management. Patient characteristics and operation rates were compared between the gastrografin challenge success group and failure group, and operation rates and length of hospital stay were compared between the gastrografin challenge group and "non-challenge" group.
\end{abstract}

Results: During the study period, 21 patients with ASBO underwent the gastrografin challenge. The challenge was successful in 17 patients where the bowel obstruction was resolved without the need for surgery. Among patients who failed the challenge, 2 patients underwent adhesiolysis and 2 patients were able to progress their diet avoiding surgery. In patients who underwent surgery ( $n=$ 2 ), the length of hospital stay was significantly shorter in the gastrografin challenge group compared with the "non-challenge" group sub analysis ( $n=13$ cases; 10.5 vs. 20 days, $p=0.038$ ), indicating that the gastrografin challenge assisted rapid decision-making for surgery. No adverse events were reported for the 21 gastrografin challenges.

Conclusion: In patients with ASBO, the gastrografin challenge is an accurate, safe method to determine the need for surgery. In addition, the gastrografin challenge may reduce the length of stay in patients who required surgery for ASBO resolution.

Keywords: ileus, general surgery, adhesion, gastrografin

\section{Introduction}

Adhesive small bowel obstruction (ASBO) is one of the most important presentations in gastrointestinal emergencies [13], with previous abdominal surgery being a major etiology [4]. Although the use of anti-adhesive barriers and an increase in the use of laparoscopic surgery have decreased adhesionrelated complications [5,6], a recent epidemiologic study has reported that 1 in 4 patients who undergo open or laparoscopic abdominal surgery is readmitted for causes related to adhesions [6]. Furthermore, ASBO has a high recurrence rate of up to $20 \%$ within 5 years, either with conservative management or surgical treatment [7].

ASBO accompanying small bowel strangulation or total obstruction require immediate surgery [8]. However, the decision-making can be challenging when physical examination, laboratory test results or computed tomography findings, are not distinct. Old practice guidelines $[9,10]$ suggest selecting patients and providing conservative care. However, the length of time to resolve is debated, with some studies suggesting waiting for more than 7 days [11], while other studies report that a delay of more than 4 days results in an increased length of hospital stay and incidence of death [12].

The gastrografin challenge is commonly performed to 
diagnose and aid decision-making in patients with ASBO to continue with conservative management or pursue operative management. Gastrografin is a safe, water-soluble contrast medium used with radiography to evaluate the integrity of the intestine. The possible therapeutic effects of gastrografin are unclear. Several studies have suggested that the use of gastrografin decreases the length of hospital stay and reduces the need for surgery [13-16]. However, some randomizedcontrolled studies, have suggested that the use of gastrografin only decreases the length of hospital stay, and does not affect mortality or the need for surgery $[17,18]$. Although the therapeutic effect of gastrografin is questionable, gastrografin has been proven to be a safe, and accurate predictor of the need for surgery, and the recently revised guidelines from the World Society of Emergency Surgery Adhesive Small Bowel Obstruction Working Group recommends using the gastrografin challenge for decision-making [19].

With the transition of contemporary abdominal surgery to minimal invasiveness and use of various chemicals, this retrospective study aimed to demonstrate the feasibility of the gastrografin challenge for adhesive bowel obstruction in a tertiary medical center, and evaluate the institution protocol for such cases.

\section{Materials and Methods}

A retrospective study was performed by reviewing medical data from a single tertiary referral center (Samsung Medical Center). This study was approved by the institutional review board of Samsung Medical Center (Approval no.: 2020-08-115001 ) which waived the need for informed consent owing to the retrospective nature of the study.

The medical records of all patients who were admitted to the Department of General Surgery (GS) from September 2018 to August 2019 with a diagnosis related to ASBO were reviewed. Using the International Classification of Diseases 10th revision, a diagnosis related to ASBO included paralytic ileus (K56.0), intestine adhesions (bands) with obstruction (K56.5), other unspecified intestinal obstruction (K56.6), and ileus unspecified (K56.7), were considered diagnosis related to ASBO. Patients who underwent the gastrografin challenge were included in the study population.

Pediatric patients ( $<18$ years), patients admitted without visiting the emergency room (ER), and patients who required an emergency operation due to small bowel strangulation, complete bowel obstruction, or a septic condition indicating peritonitis were excluded from the study.

The gastrografin challenge was performed by many physicians, mainly on the acute care surgery team, a subdivision of GS managing GS emergencies and care on the
Intensive Care Unit. Patients who were directly referred to the surgeon from a previous operation were mostly managed with traditional methods (without using the gastrografin challenge).

Data on basic patient characteristics (such as age, sex, history of previous abdominal operation, and days from previous operation to ER admission), laboratory blood test results (such as complete blood count with differential counts; serum lactate level; C-reactive protein level, and erythrocyte sedimentation rate), and imaging study findings (such as simple abdominal radiography, and abdominal and pelvic computed tomography scans) were collected using an electronic medical record system.

\section{Protocol for the gastrografin challenge}

The protocol for the gastrografin challenge (Figure 1) is as follows: decompression of the intestine with a nasogastric (NG) tube for 2 hours before administration of gastrografin; administration of $100 \mathrm{~mL}$ of gastrografin with $50 \mathrm{~mL}$ of water through the NG tube; and clamping of the NG tube with simple abdominal radiographs taken $1,2,4,8,12$, and 24 hours after gastrografin administration.

If the contrast reached the ascending colon within 24 hours of gastrografin administration, the gastrografin challenge was considered successful. If the contrast was not visible in the colon within 24 hours, the gastrografin challenge was regarded as a failure. When the gastrografin challenge succeeds, resolution of ASBO is expected and conservative management can be continued. In the case of failure, surgical treatment is considered.

If vomiting occurs at any stage after NG tube clamping, the tube is declamped and the gastrografin challenge is regarded as a failure. In cases of aggravating symptoms or abdominal

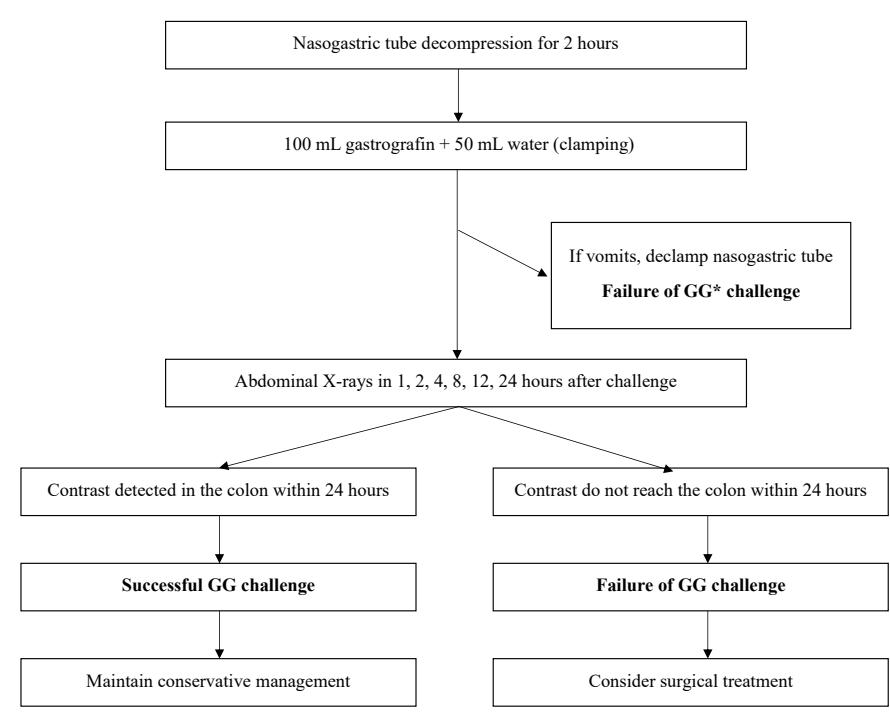

Figure 1. The gastrografin challenge protocol. $\mathrm{GG}=$ gastrografin. 
physical examination, or when the progression of peritonitis is suspected, the gastrografin challenge is suspended and surgical treatment is considered at any stage.

\section{Statistical analysis}

The feasibility of the gastrografin challenge protocol was verified by reviewing how much success in the gastrografin challenge led to the resolution of ASBO without surgery. Failure of the gastrografin challenge ultimately led to surgical treatment. Therefore, the primary study outcome was how much success in the gastrografin challenge predicted unnecessary surgery and failure predicted the need for surgery in patients with ASBO. The gastrografin challenge success group and failure group were compared using Student t test for numeric data and the chi-square test for categorical data. Secondary study outcomes included length of hospital stay and operation rates in the gastrografin challenge group (patients who underwent the gastrografin challenge) and the "nongastrografin challenge" group (patients who did not undergo the gastrografin challenge group) to determine the therapeutic effect of the gastrografin challenge. The length of hospital stay was compared between groups using the Mann-Whitney test, and the operation rates were compared between groups using the Fisher's exact test. In addition, the time to resolution after the gastrografin challenge, readmission rate, and safety of the gastrografin challenge were also investigated.

\section{Results}

During the study period, there were 125 patients ( 140 cases) admitted to hospital with a diagnosis related to ASBO. Among the 140 cases, 7 cases pertained to patients aged $<18$ years, 7 cases pertained to patients admitted without visiting the ER, and 12 cases requiring an emergency operation were

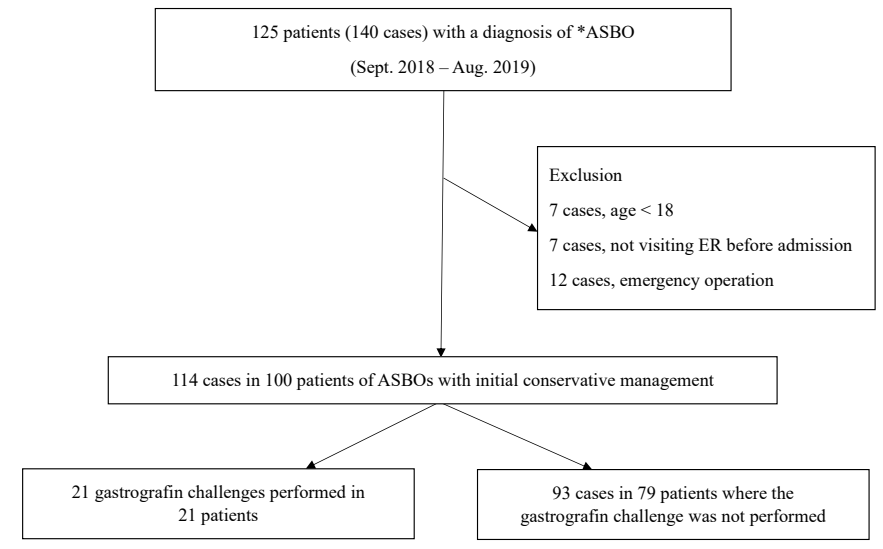

Figure 2. The scheme for patient selection. ASBO = adhesive small bowel obstruction. excluded from the study. Among 114 ASBO cases that were conservatively managed, the gastrografin challenge was performed in 21 cases (Figure 2).

Among the 21 patients (21 cases) who underwent the gastrografin challenge, 19 patients had a history of a previous operation, primarily colorectal surgery ( 8 of $19,42.1 \%$ ). The mean time between the previous operation and hospital visit was 88 months.

The gastrografin challenge was successful in 17 (81.0\%) of 21 patients, and none of them underwent abdominal exploration (Table 1). Among the 4 patients who failed the challenge, 2 patients underwent adhesiolysis, and 2 patients were able to build up their diet without surgery. There were no statistical differences in the patient characteristics between the gastrografin challenge success group and failure group.

In the success group, the mean time from previous operation to hospital visit was 74 months, and 7 patients had a history of previous admission due to small bowel obstruction. Laboratory blood test results revealed normal findings (Table 1 ).

Among the 4 patients who failed the gastrografin challenge, 2 patients showed symptomatic relief during the consideration of operation. Contrast was eventually visible in the colon in these cases ( 40 hours and 48 hours after the gastrografin challenge), and patients were able to progress their diet without the need for an operation.

Two patients who underwent operation presented with abdominal pain, nausea, and vomiting on the first examination in the ER. Vital signs and physical examination did not show any evidence of peritonitis. The gastrografin challenge was performed on Day 4 and Day 5 after admission. Following the failure of the challenge, adhesiolysis was performed, and patients were able to progress their diet and were discharged without complications.

In the gastrografin challenge success group, the mean time from the ER visit to the gastrografin challenge was $4.5 \pm 3.1$ days. The mean time between the gastrografin challenge and contrast detection in the colon was $5.0 \pm 6.6$ hours. The longest time for the contrast to be detected in the colon was 24 hours. Hospital discharge was decided based on progression of diet, and the mean length of hospital stay was $9.1 \pm 4.0$ days (Table 2 ). After hospital discharge, 4 patients were readmitted due to complications related to adhesions, of whom 3 patients underwent surgery (Table 1).

Regarding the therapeutic effect of the gastrografin challenge, although not statistically significant, the length of hospital stay was shorter in the gastrografin challenge group compared with the "non-challenge" group. In addition, the operation rate did not differ between gastrografin challenge group and "non-challenge" group. However, in the subgroup analysis, the length of hospital stay was significantly shorter in patients who underwent surgery in the gastrografin challenge group 
Table 1. Comparison of patient characteristics and operation rates between the gastrografin challenge group and the failure group.

\begin{tabular}{|c|c|c|c|}
\hline & $\begin{array}{l}\text { Success group } \\
\quad(n=17)\end{array}$ & $\begin{array}{l}\text { Failure group } \\
\qquad(n=4)\end{array}$ & $p$ \\
\hline Age (mean, y) & 68 & 67.75 & $0.97^{*}$ \\
\hline Female (\%) & $7(41.12)$ & $2(50.0)$ & $0.91^{\dagger}$ \\
\hline Operation history (\%) & $16(94.12)$ & $3(75.0)$ & $0.24^{\dagger}$ \\
\hline Colorectal & 6 & 2 & \\
\hline Hepatobiliary & 2 & 1 & \\
\hline Stomach & 3 & & \\
\hline OBGY & 2 & & \\
\hline Aorta & 2 & & \\
\hline Small bowel & 1 & & \\
\hline Postoperative time (mo) & 74.81 & 123.33 & $0.49^{*}$ \\
\hline \multicolumn{4}{|l|}{ No. ileus } \\
\hline 0 & 12 & 3 & \\
\hline 1 & 1 & & \\
\hline 2 & 2 & 1 & \\
\hline$>3$ & 4 & & \\
\hline \multicolumn{4}{|l|}{ Laboratory results } \\
\hline $\mathrm{WBC}\left(\times 10^{3} / \mathrm{mL}\right)$ & 7.83 & 9.47 & $0.50^{*}$ \\
\hline Lactate $(\mathrm{mg} / \mathrm{dL})$ & 1.76 & 2.31 & $0.49^{*}$ \\
\hline $\mathrm{CRP}(\mathrm{mg} / \mathrm{dL})$ & 1.71 & 1.64 & $0.96^{*}$ \\
\hline OP performed & 0 & 2 & \\
\hline Readmission & 4 & 1 & \\
\hline Reoperation & 3 & 0 & \\
\hline
\end{tabular}

* Student $t$ test.

$\dagger$ Chi-square test.

$\mathrm{CRP}=\mathrm{c}$-reactive protein; $\mathrm{OBGY}=$; $\mathrm{OP}=$ operation; $\mathrm{WBC}=$ white blood cell count.; $\mathrm{OBGY}=$ obstetrics and gynecology.

Table 2. Profile of the gastrografin challenge success group.

\begin{tabular}{|c|c|c|c|}
\hline \multicolumn{4}{|c|}{$\begin{array}{l}\text { GG success group } \\
\qquad(n=17)\end{array}$} \\
\hline Time & Mean \pm SD & $\begin{array}{c}\text { Range } \\
\text { maximum }\end{array}$ & $\begin{array}{c}\text { Range } \\
\text { minimum }\end{array}$ \\
\hline Admission until GG challenge (d) & $4.53 \pm 3.09$ & 1 & 13 \\
\hline Detection of contrast after challenge (h) & $5.00 \pm 6.62$ & 1 & 24 \\
\hline Hospital stay (d) & $9.12 \pm 4.01$ & 2 & 17 \\
\hline
\end{tabular}

GG = gastrografin.

( $n=2$ ) compared with the "non-challenge" group ( $n=13$; 10.5 vs. 25.0 days, $p=0.038$ ), indicating that the gastrografin challenge may have assisted rapid decision-making for surgery
(Table 3).

No adverse events were reported in all 21 gastrografin challenges. 
Table 3. Comparison between the gastrografin challenge group and the "non-gastrografin challenge" group.

\begin{tabular}{lccc}
\hline & $\begin{array}{c}\text { GG challenge } \\
(n=21)\end{array}$ & $\begin{array}{c}\text { "Non-GG challenge" } \\
(n=93)\end{array}$ & $0.267^{*}$ \\
\hline Hospital stay (d) & $9.29 \pm 3.96$ & $10.80 \pm 10.03$ & $1.00^{\dagger}$ \\
Operations (\%) & $2(14.3)$ & $13(14.0)$ & $0.038^{\ddagger}$ \\
$\begin{array}{l}\text { Hospital stay for surgical patients, } \\
\text { median } \pm \text { IQR (d) }\end{array}$ & $10.5 \pm 5.0$ & $28.0 \pm 25.0$ & \\
\hline
\end{tabular}

Data are presented as $n(\%)$ or mean \pm SD.

* Student $\mathrm{t}$ test.

$\dagger$ Fisher's exact test.

$\ddagger$ Mann-Whitney test.

$\mathrm{IQR}$ = interquartile range; $\mathrm{GG}=$ gastrografin .

\section{Discussion}

This study investigated the feasibility of the gastrografin challenge in patients with ASBO, with protocols modified for contemporary ER circumstances. Several studies have already reported the safety and usefulness of gastrografin $[16-18,20]$. Recently, the guideline by the World Society of Emergency Surgery Adhesive Small Bowel Obstruction Working Group was updated, which recommends the use of gastrografin challenge for surgical decision-making in cases of ASBO [19].

However, the guidelines for ASBO are not actively applied in clinical settings, especially in the Republic of Korea, with few studies reporting cases in which the gastrografin challenge was performed. The traditional method of decompression and surgical observation is the main treatment in the Republic of Korea. This study showed how the gastrografin challenge protocol can be adopted in clinical settings, especially in a tertiary referral center of the Republic of Korea.

In this study, 17 patients with ASBO in whom the gastrografin challenge was successfully performed did not require surgery for resolution of the obstruction. Of the 4 patients who failed the gastrografin challenge, 2 patients underwent surgery. In the other 2 patients who did not require surgery, gastrografin was observed in their colons eventually. A comparison of patients who received the gastrografin challenge with those who did not receive the challenge, revealed that the gastrografin challenge reduced the length of hospital stay in patients who required surgery to relieve the obstruction. In all patients who underwent the gastrografin challenge, no adverse events were reported.

This study showed that the success of the gastrografin challenge can strongly predict the resolution of ASBO without surgery. One previous study reported 96\% sensitivity and 98\% specificity for ASBO resolution without surgery when contrast media was observed in the colon [16]. In our study, operation was unnecessary in all patients in whom the challenge was successful.
In the gastrografin challenge failure group, only half of the patients underwent surgery. The other half of the patients did not require surgery; however, gastrografin was eventually detected in the colon within 48 hours. Therefore, if the protocol is modified for a longer observation period, greater specificity may be achieved.

In the gastrografin challenge success group, contrast detection in the colon was observed on average within 5 \pm 6.6 hours. Regarding the timing for contrast detection, a study claimed 6 hours should be sufficient [21], while other studies suggested waiting up to 72 hours $[22,23]$. The Bologna guideline by the ASBO working group suggests considering surgical treatment when contrast media does not reach the colon within 36 hours of the challenge [19]. Although 5 hours was the mean time for detection in our study, the 24 hours limit in the protocol seems rather short, considering the findings of other studies and patients who did not require surgery, but failed the gastrografin challenge.

Although the sample number is low and further investigation is required, the results of this study also suggest that the gastrografin challenge can shorten the length of hospital stay, especially in patients with ASBO who require surgery. The necessity of surgery did not differ between the gastrografin challenge group and "non-challenge" group, however, gastrografin challenge failure seems to aid in the early decisionmaking in patients with ASBO requiring surgery. Regarding the therapeutic effect of the gastrografin challenge, several previous meta-analyses have shown controversial results. Two studies claimed that the use of gastrografin decreases the length of hospital stay by allowing early decision-making, and reduces the need for surgery by decreasing small bowel edema and increasing small bowel contractility $[16,20]$. However, other meta-analyses reported that the use of gastrografin only decreases the length of hospital stay and does not affect mortality or the need for surgery $[17,18]$.

Regarding the need for surgery, approximately $14 \%$ patients with ASBO underwent surgery in our study, both in the gastrografin challenge group and "non-challenge" group. 
However, in previous studies, $20-30 \%$ [1,24] to $45 \%$ [6] patients with ASBO required surgery. A larger portion of laparoscopic surgery and active adoption of anti-adhesive barriers may have affected the results. Laparoscopic surgery, although mainly adopted because of its minimal invasiveness, reduces adhesion formation and adhesion-related complications $[6,25]$. The efficacy of anti-adhesive barriers has been proven by highquality evidence-based studies $[5,26]$. Further investigations are necessary to evaluate the ratio of laparoscopic surgery and the use of anti-adhesive barriers. With lower rates of surgery in patients with ASBO, protocols using the gastrografin challenge are expected to gain more value in the future.

The limitations of this study include its retrospective nature and the small number of cases. However, with increased experience of performing the gastrografin challenge in patients with ASBO and the safety of the procedure, further studies are expected such as active adoption of the gastrografin challenge, a shorter time to gastrografin challenge after admission, and less abdominal radiographs. In addition, efficient protocols for the gastrografin challenge will be established and statistically robust results will be achieved by including more cases.

In conclusion, the gastrografin challenge is a safe method for decision-making to determine whether surgical treatment in patients with ASBO should be performed. Additionally, the gastrografin challenge may aid in reducing the length of hospital stay in such patients.

\section{Conflicts of Interest}

Chi-Min Park has been the editor in chief of the Journal of Acute Care Surgery since January 2011, but had no role in the decision to publish this original article. No other potential conflict of interest relevant to this article was reported.

\section{Funding}

The abstract for this study won the Young Investigator award and was published in the Annual Congress of the Korean Surgical Society. However, this article has not been submitted for publication in other journals and has not been published.

\section{References}

[1] ten Broek RP, Issa Y, van Santbrink EJ, Bouvy ND, Kruitwagen RF, Jeekel J, et al. Burden of adhesions in abdominal and pelvic surgery: Systematic review and met-analysis. BMJ 2013;347:f5588.

[2] Patel R, Borad NP, Merchant AM. Comparison of outcomes following laparoscopic and open treatment of emergent small bowel obstruction: An 11-year analysis of ACS NSQIP. Surg Endosc 2018;32(12):4900-11.

[3] Miller G, Boman J, Shrier I, Gordon PH. Etiology of small bowel obstruction. Am J Surg 2000;180(1):33-6.

[4] Lee MJ, Sayers AE, Drake TM, Hollyman M, Bradburn M, Hind D, et al. UK- based, multisite, prospective cohort study of small bowel obstruction in acute surgical services: National Audit of Small Bowel Obstruction (NASBO) protocol. BMJ Open 2017;7(10):e016796.

[5] van Steensel S, van den Hil LCL, Schreinemacher MHF, Ten Broek RPG, van Goor H, Bouvy ND. Adhesion awareness in 2016: An update of the national survey of surgeons. PLoS One 2018;13(8):e0202418.

[6] Krielen P, Stommel MWJ, Pargmae P, Bouvy ND, Bakkum EA, Ellis H, et al. Adhesion-related readmissions after open and laparoscopic surgery: A retrospective cohort study (SCAR update). Lancet 2020;395(10217):33-41.

[7] Foster NM, McGory ML, Zingmond DS, Ko CY. Small bowel obstruction: A population-based appraisal. J Am Coll Surg 2006;203(2):170-6.

[8] Choi HK, Chu KW, Law WL. Therapeutic value of gastrografin in adhesive small bowel obstruction after unsuccessful conservative treatment: A prospective randomized trial. Ann Surg 2002;236(1):1-6.

[9] Maung AA, Johnson DC, Piper GL, Barbosa RR, Rowell SE, Bokhari F, et al. Evaluation and management of small-bowel obstruction: An Eastern Association for the Surgery of Trauma practice management guideline. J Trauma Acute Care Surg 2012;73(5 Suppl 4):S362-9.

[10] Di Saverio S, Coccolini F, Galati M, Smerieri N, Biffl WL, Ansaloni L, et al. Bologna guidelines for diagnosis and management of adhesive small bowel obstruction (ASBO): 2013 update of the evidence-based guidelines from the world society of emergency surgery ASBO working group. World J Emerg Surg 2013;8(1):42.

[11] Bickell NA, Federman AD, Aufses AH, Jr. Influence of time on risk of bowel resection in complete small bowel obstruction. J Am Coll Surg 2005;201(6):847-54

[12] Schraufnagel D, Rajaee S, Millham FH. How many sunsets? Timing of surgery in adhesive small bowel obstruction: A study of the Nationwide Inpatient Sample. J Trauma Acute Care Surg 2013;74(1):181-7; discussion 187-9.

[13] Assalia A, Schein M, Kopelman D, Hirshberg A, Hashmonai M. Therapeutic effect of oral Gastrografin in adhesive, partial small-bowel obstruction: A prospective randomized trial. Surgery 1994;115(4):433-7.

[14] Chen SC, Chang KJ, Lee PH, Wang SM, Chen KM, Lin FY. Oral urografin in postoperative small bowel obstruction. World J Surg 1999;23(10):1051-4.

[15] Biondo S, Parés D, Mora L, Martí Ragué J, Kreisler E, Jaurrieta E. Randomized clinical study of Gastrografin administration in patients with adhesive small bowel obstruction. Br J Surg 2003;90(5):542-6.

[16] Branco BC, Barmparas G, Schnüriger B, Inaba K, Chan LS, Demetriades D. Systematic review and meta-analysis of the diagnostic and therapeutic role of water-soluble contrast agent in adhesive small bowel obstruction. Br J Surg 2010;97(4):470-8.

[17] Abbas S, Bissett IP, Parry BR. Oral water soluble contrast for the management of adhesive small bowel obstruction. Cochrane Database Syst Rev 2007;2007(3):CD004651.

[18] Koh A, Adiamah A, Chowdhury A, Mohiuddin MK, Bharathan B. Therapeutic Role of Water-Soluble Contrast Media in Adhesive Small Bowel Obstruction: A Systematic Review and Meta-Analysis. J Gastrointest Surg 2020;24(2):473-83.

[19] Ten Broek RPG, Krielen P, Di Saverio S, Coccolini F, Biffl WL, Ansaloni L, et al. Bologna guidelines for diagnosis and management of adhesive small bowel obstruction (ASBO): 2017 update of the evidence-based guidelines from the world society of emergency surgery ASBO working group. World J Emerg Surg 2018;13:24.

[20] Ceresoli M, Coccolini F, Catena F, Montori G, Di Saverio S, Sartelli M, et al. Water-soluble contrast agent in adhesive small bowel obstruction: A systematic review and meta-analysis of diagnostic and therapeutic value. Am J Surg 2016;211(6):1114-25.

[21] Brochwicz-Lewinski MJ, Paterson-Brown S, Murchison JT. Small Bowel Obstruction-The Water-soluble Follow-through Revisited. Clin Radiol 2003;58(5):393-7.

[22] Di Saverio S, Catena F, Ansaloni L, Gavioli M, Valentino M, Pinna AD. Water-Soluble Contrast Medium (Gastrografin) Value in Adhesive Small Intestine Obstruction (Asio): A Prospective, Randomized, Controlled, Clinical Trial. World J Surg 2008;32(10):2293-304.

[23] Galardi N, Collins J, Friend K. Use of Early Gastrografin Small Bowel Follow-through in Small Bowel Obstruction Management. Am Surg 2013;79(8):794-6.

[24] Musiienko AM, Shakerian R, Gorelik A, Thomson BN, Skandarajah AR. Impact of introduction of an acute surgical unit on management and outcomes of small bowel obstruction. ANZ J Surg 2016;86(10):831-5.

[25] Stommel MWJ, Ten Broek RPG, Strik C, Slooter GD, Verhoef C, Grünhagen DJ, et al. Multicenter Observational Study of Adhesion Formation After Open-and Laparoscopic Surgery for Colorectal Cancer. Ann Surg 2018;267(4):743-8.

[26] Ten Broek RPG, Stommel MWJ, Strik C, van Laarhoven C, Keus F, van Goor $\mathrm{H}$. Benefits and harms of adhesion barriers for abdominal surgery: A systematic review and meta-analysis. Lancet 2014;383(9911):48-59. 